\title{
Percentage Change on FDG-PET/CT Predicts Complete Response to Neoadjuvant Radiochemotherapy in Esophageal Cancer
}

\author{
Esther Jimenez-Jimenez ${ }^{*}{ }^{\circledR}$, Irene Ortiz², Neus Aymar², Raquel Roncero², Pedro Mateos ${ }^{3}$, \\ Marta Gimenez ${ }^{4}$, Jose Pardo ${ }^{2}$, Sebastià Sabater ${ }^{1}$
}

${ }^{1}$ Radiation Oncology Department, Complejo Hospitalario Universitario, de Albacete, Spain

${ }^{2}$ Radiation Oncology Department, Hospital Universitari Son Espases, Palma de Mallorca, Spain

${ }^{3}$ Medical Physics Department, Hospital Universitari Son Espases, Palma de Mallorca, Spain

${ }^{4}$ Nuclear Medicine Department, Hospital Universitari Son Espases, Palma de Mallorca, Spain

Email: *estherjj@hotmail.com

How to cite this paper: Jimenez-Jimenez, E., Ortiz, I., Aymar, N., Roncero, R., Mateos, P., Gimenez, M., Pardo, J. and Sabater, S. (2019) Percentage Change on FDG-PET/CT Predicts Complete Response to Neoadjuvant Radiochemotherapy in Esophageal Cancer. International Journal of Clinical Medicine, 10, 531-542.

https://doi.org/10.4236/ijcm.2019.1010043

Received: September 23, 2019

Accepted: October 19, 2019

Published: October 22, 2019

Copyright $\odot 2019$ by author(s) and Scientific Research Publishing Inc. This work is licensed under the Creative Commons Attribution International License (CC BY 4.0).

http://creativecommons.org/licenses/by/4.0/

\begin{abstract}
Objective: We evaluated whether the changes in FDG-PET/CT uptake between pre/and post-treatment might predict a complete response in esophageal cancer (EC). Patients and Methods: Fifty-six patients with 2 PET-CTs studies were evaluated retrospectively. Images were evaluated qualitatively and semiquantitatively (SUVs). Patients were classified as persistence of disease, complete metabolic response and unspecific FDG uptake. The SUVmax values and percentages of change in SUV were measured. Results: A significant difference was found between the persistence group and the non-persistence group regarding the median percentage change in SUVtumor $(72.95 \%$ vs. $54.12 \% ; \mathrm{p}=0.04)$ and regarding the percentage change in SUVnode $(89.91 \%$ vs. $59.91 \%, p=0.04)$. In patients treated with radiochemotherapy (RCTX), a significant difference was found between the persistence group and the no persistence group regarding the percentage change in SUVtumor $(58.02 \%$ vs. $78.59 \%)$. Overall survival rate was related to the percentage of change in the SUVtumor. The group of $\geq 75 \%$ of change SUVtumor showed a median survival of 37.32 months (IC: $95 \%=49.93-24.70$ ) and the group of $<75 \%$ of change SUVtumor showed a median survival of 18.39 months (IC: $95 \%=$ $25.14-11.65)(p=0.04)$. In patients with metastatic relapse, a significant difference was found regarding the percentage change in SUVnode $(94.63 \%$ vs. $74.09 \%)$. Conclusion: Our study provides evidence that the percentage change in the SUVmax is a predictor of the response to neoadjuvant treatment in patients with EC. When SUVmax decreases by $72.95 \%$ or more, the patient is
\end{abstract}


likely a complete responder.

\section{Keywords}

PET/CT, Esophageal, Response, SUV, Percentage

\section{Introduction}

Esophageal cancer (EC) is the sixth leading cause of cancer-related mortality worldwide [1] and most patients have locally advanced disease at diagnosis. Currently, the standard of care for patients with resectable locally advanced esophageal or esophagogastric junctional cancer is neoadjuvant radiochemotherapy (RCTX) or chemotherapy (CTX) followed by surgical resection [2] [3]. However, for squamous cell carcinoma, two randomized trials have reported no survival benefits of surgery [4] [5] and for this reason, there is a growing interest in selecting patients who could be treated by non-invasive tests rather than surgery.

Nowadays, the value of FDG-PET/CT for restaging after induction treatment is not clear and international guidelines differ in their recommendations. Limited studies suggest that PET/CT imaging detects distant metastases following induction RCTX [6] and some centers routinely order a post-induction PET/CT approximately four weeks after the completion of treatment as a method to assess for distant metastatic disease. In addition, post-induction-therapy FDG-PET provides information on the metabolic response in the primary tumor that may be clinically useful in the selection of subsequent therapy [7]. However, others have failed to find a correlation between the post-RCTX SUV on PET and pathologic response at the time of resection [8].

According to current evidence, the main use of PET-CT in the revaluation of EC may be to recognize which patients are not responding to induction CTX [9]. However, there is limited evidence according to the role of PET-CT after RCTX. For this reason, the aim of this study was to assess the role of FDG-PET/CT in response evaluation to neoadjuvant RCT in the EC and determine whether the changes in FDG-PET/CT uptake between pre/and post-induction treatment could be related to the persistence or not of tumor.

\section{Patients and Methods}

\subsection{Patients}

Fifty-six patients with locally advanced EC treated between April 2013 and November 2016 were evaluated retrospectively following approval by the local ethics committee. All patients were required to have staging with a physical examination, endoscopic/ultrasonography and cervical/thoracic/abdominal CT. The eighth edition of the TNM staging was used. All of them had at least 2 FDG-PET/CT studies, one before RCTX with PET/CT1 as a baseline and the 
other after treatment (PET/CT2). No patient had metastatic disease at the time of diagnosis. The images were confirmed by histopathology.

\subsection{FDG-PET/CT STAGING (PET/CT1)}

Image acquisition procedures have been described elsewhere [10]. In summary, all patients fasted for at least $6 \mathrm{~h}$ before the FDG-PET/CT examination. They were injected with $4 \mathrm{MBq} / \mathrm{kg}$ body weight of $18 \mathrm{FDG}$ and then rested for about 1 $\mathrm{h}$ in a quiet room before imaging. CT acquisition parameters were: $100 \mathrm{kV}, 80$ $250 \mathrm{~mA}$, slice thickness of $2.5 \mathrm{~mm}$. It was used both for the attenuation correction of PET data and to locate the FDG uptake in PET images. PET scanning was performed covering the same axial range for 2 min per bed position (total of 3 5 bed positions). Both PET and CT acquisition were performed during free breathing. Data were reconstructed using an ordered subset expectation maximization (OSEM), 3D algorithm (3D Iterative) and attenuation correction derived from CT data. The average acquisition time of the images was between 12 16 minutes. The maximal standardized tumor uptake values (SUVmax) were normalized by lean body mass (LBM). The FDG-PET image was normalized to the physiological FDG uptake in the liver and, after identifying the primary tumor as a region of interest (ROI), the SUVmax was obtained from the most metabolically active lesion.

\subsection{Treatment and Follow-Up}

Patients underwent RCTX or neoadjuvant CTX. The radiotherapy (RTX) was delivered using a three-dimensional conformal technique. Median radiation dose was 50.4 Gy. The gross tumor volume (GTV) was delineated according to Visual analysis of nuclear medicine physicians [11] and the clinical target volume (CTV) according to the ESTRO and EORTC-RTOG recommendations [12]. It consisted of the primary lesions and regional nodal regions with a 3 to $5 \mathrm{~cm}$ craniocaudal and 1 to $2 \mathrm{~cm}$ circumferential margin. The CTX treatment scheme was CDDP-5FU or EOX according to histology. Surgical resection was performed on 16 patients. The decision on local resectability was taken by a multidisciplinary committee and depended on tumor infiltration into neighboring structures, distant metastases and absence/presence of uptake of FDG-PET/CT. The histopathological response was measured according to the Mandard tumor regression grade scoring system [13]. Patients were followed up every 3 - 6 months. Locoregional control was defined as "time until proven recurrence". Overall survival was defined as "time until death".

\subsection{FDG-PET/CT Re-Evaluation (PET/CT2)}

All patients were re-evaluated by PET/CT2, in the same nuclear medicine department and PET/CT2 was carried out 3 months after RCTX. The response by $\mathrm{PET} / \mathrm{CT}$ was measured in absolute terms and as a percentage reduction in SUVmax. No other assessment parameters were used (such as metabolic tumor 
volume, total lesion glycolysis or SUVmean) because they are not usually used in clinical practice.

\subsection{Image Interpretation and Analysis}

Image analysis procedures have already been described [14]. The PET images were evaluated qualitatively (visual inspection) and semiquantitatively (SUVs). PET/CT2 scans were interpreted and correlated with clinical information and the initial PET/CT1. Patients were classified into 3 groups: persistence of disease (PET/CT2 shows loco/regional disease or progression), complete metabolic response (SUVmax on PET/CT2 $\leq 3$ ) and unspecific FDG uptake. A team of experienced nuclear medicine physicians and radiologists defined whether persistence or non-persistence of disease. They evaluated tumor recurrence at the anastomosis site of the resected primary tumor based on a visual analysis on the PET-CT scan. The following characteristics were used to describe FDG uptake on fused PET-CT images: the asymmetric of the uptake and the extend of FDG uptake, whereas malignant uptake tends to be focal, eccentric, and benign uptake (due to RT or reflux esophagitis) tends to be diffuse and concentric. Suspect lesions were confirmed by biopsy or by radiological and clinical follow-up. Patients who underwent surgery were not classified in these groups.

The SUVmax values of the esophageal tumor and lymph nodes on PET/CT images were collected. Percentages of change in SUV were calculated as $(\mathrm{PET} / \mathrm{CT} 1 \mathrm{SUV}-\mathrm{PET} / \mathrm{CT} 2 \mathrm{SUV}) \times 100 / \mathrm{PET} / \mathrm{CT} 1 \mathrm{SUV}$. There are studies that have divided these into subcategories according to percentage change but there are no generally accepted threshold values [15] [16]. In the present study, all uptakes were divided into two groups according to the percentage of change in the SUV ( $\geq 75 \%$ and $<75 \%)$. We used this value because we found the persistence group had a percentage of change in SUV tumor of $72.95 \%$.

\subsection{Statistical Analysis}

Overall survival (OS) and progression-free survival (PFS) were calculated by Kaplan-Meier survival analysis, and comparison between groups was made by the log-rank test. Univariate and multivariate Cox proportional models were used and a value of $\mathrm{p}<0.05$ was considered to be statistically significant. SPSS v. 22.00 was used for statistical analysis.

\section{Results}

\subsection{Patient Characteristics}

Median follow-up of patients was 20.4 months (range: 4.2 - 56.5 weeks). Figure 1 shows a profile of this studyin which 56 patients were assessed (men $=47$, women $=9$, with median age 62.7 years $( \pm 8.2)$. The patients' characteristics are provided in Table 1 . Sixteen patients (28.6\%) underwent surgery according to the response and 40 patients (71.4\%) were treated without surgery. 


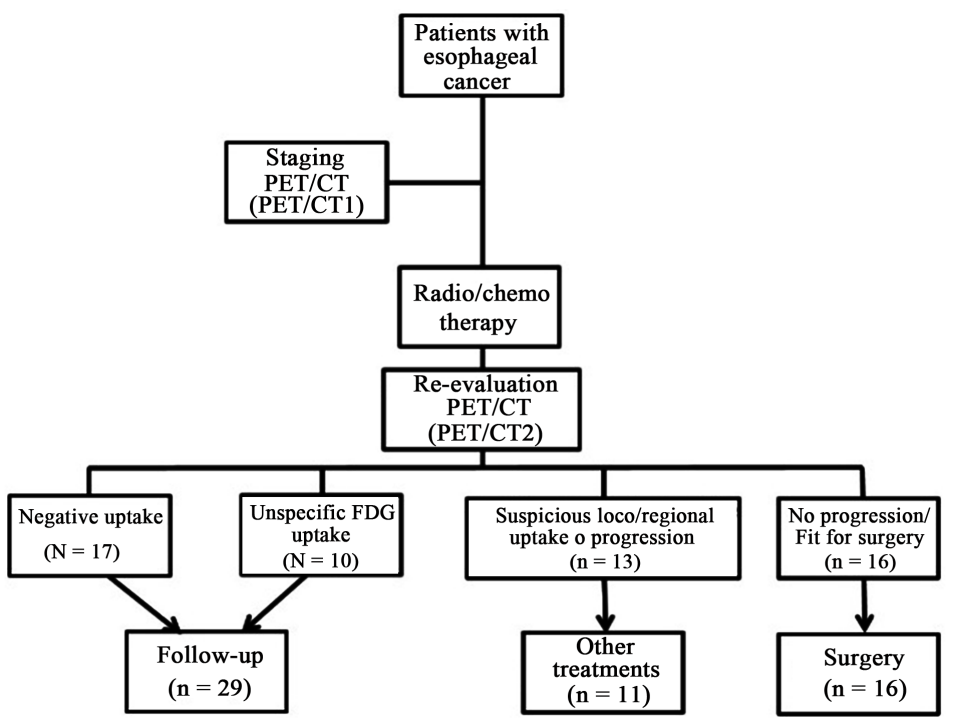

Figure 1. Study population and design.

Table 1. Patients' characteristics and findings on PET/CT1.

\begin{tabular}{|c|c|}
\hline Characterisits & Value \\
\hline Numer of patients & 56 \\
\hline Age (years) (median \pm DS) & $62.7 \pm 8.2$ \\
\hline \multicolumn{2}{|l|}{ Gender } \\
\hline Male & $47(83.9 \%)$ \\
\hline Female & $9(16.1 \%)$ \\
\hline \multicolumn{2}{|l|}{ Location } \\
\hline Cervical & $11(19.6 \%)$ \\
\hline Thoracic & $20(35.7)$ \\
\hline Lower & $25(44.6 \%)$ \\
\hline \multicolumn{2}{|l|}{ Histology } \\
\hline Squamous & $42(75 \%)$ \\
\hline Adenocarcinoma & $13(23.2 \%)$ \\
\hline \multicolumn{2}{|l|}{ Grade } \\
\hline G1-2 & $7(12.5 \%)$ \\
\hline G3-4 & $17(30.4 \%)$ \\
\hline Unknown & $32(57.1 \%)$ \\
\hline \multicolumn{2}{|l|}{ Treatment } \\
\hline Radio-chemo & $47(83.9 \%)$ \\
\hline Chemo & $9(16.1 \%)$ \\
\hline \multicolumn{2}{|l|}{ Tumor +/- (PET/CT1) } \\
\hline PET-T+ & $55(98.2 \%)$ \\
\hline PET-T- & $1(1.8 \%)$ \\
\hline \multicolumn{2}{|l|}{ Node +/- (PET/CT1) } \\
\hline PET-N+ & $21(37.5 \%)$ \\
\hline PET-N- & $35(62.5 \%)$ \\
\hline \multicolumn{2}{|l|}{ Number involved nodes } \\
\hline No & $19(33.9 \%)$ \\
\hline N1 (1 - 2 nodes) & $27(48.2 \%)$ \\
\hline $\mathrm{N} 2$ (3 - 6 nodes) & $10(17.9 \%)$ \\
\hline N3 (>7 nodes) & 0 \\
\hline
\end{tabular}




\subsection{Findings on PET/CT1}

Fourteen patients were found to have stage II disease and 42 patients had stage III. The mean SUVmax values of the esophageal tumor were 12.08 (range 0 30.2 ) and 37 patients had clinically involved lymph nodes on PET/CT images: N0 (33.9\%), N1 (48.2\%), N3 (17.9\%). The mean SUVmax values of the metastatic lymph nodes were 3.94 (range 0 - 16.1). Details of findings on PET/CT1 are shown in Table 1.

\subsection{Findings on PET/CT2}

\section{- Post-treatment tumoral and node evaluation}

Thirteen patients (23.2\%) were found to have persistence of disease, a complete metabolic response was shown in 17 patients (30.4\%) and 10 patients (17.9\%) were found to have unspecific FDG uptake (with/without residual disease). 16 patients (28.6\%) underwent an operation.

The mean SUVmax values of the esophageal tumor were 3.8 (range 0 - 24.7). The mean SUVmax values of the metastatic lymph nodes were 0.8 (range 0 8.8). 13 patients had clinically involved lymph nodes on PET/CT2 images: N0 (76.8\%), N1 (19.6\%), none with N3.

All patients had a mean decrease in tumor uptake of $68.4 \%$ (SD: $33.2 \%$ ) and 55 patients had a mean decrease in node uptake of $81.6 \%$ (SD: 33.6\%), except 1 patient who had an increased node uptake. There was no correlation between the decrease in tumor or node uptake and the time between PET1 and PET2.

\section{- Changes between PET/CT1 and PET/CT2}

Patients were categorized according to follow-up into 2 groups: patients with persistence of disease or progression (Persistence) and patients with disease-free time (No persistence). Suspect lesions were confirmed. Patients who underwent surgery were excluded. These results were compared with the percentage of tumor change between both tests. A significant difference was found between the persistence group and the non-persistence group regarding the percentage change in SUVtumor (72.95\% vs $54.12 \%$ ) and post-treatment SUVtumor, and regarding the percentage change in SUVnode (89.91\% vs $59.91 \%)$ and post-treatment SUVnode (Table 2).

Secondly, we divided the patients into 2 groups according to treatment received: RCTX or only CTX. No patient was treated with the only RTX. In patients treated with RCTX, a significant difference was found between the persistence group and the no persistence group regarding the percentage change in

Table 2. Comparison between persistence and non-persistence on PET/CT imaging.

\begin{tabular}{ccccccc}
\hline & Pre-SUV tumor & $\begin{array}{c}\text { Post-SUV } \\
\text { tumor }\end{array}$ & $\begin{array}{c}\text { \% change SUV } \\
\text { tumor }\end{array}$ & Pre-SUV node & Post-SUV node & \% change SUV node \\
\hline Persistence $(+)(\mathrm{n}=13)$ & $12.5 \pm 6.15$ & $6.02 \pm 6.11$ & $54.12 \% \pm 26.68 \%$ & $5.05 \pm 3.69$ & $2.31 \pm 3.24$ & $59.91 \% \pm 47.25 \%$ \\
Persistence $(-)(\mathrm{n}=43)$ & $11.95 \pm 5.96$ & $3.13 \pm 3.43$ & $72.95 \% \pm 30.15 \%$ & $3.60 \pm 3.99$ & $0.43 \pm 1.28$ & $89.51 \% \pm 23.91 \%$ \\
& $\mathrm{p}=0.780$ & $\mathrm{p}=0.03$ & $\mathrm{p}=0.04$ & $\mathrm{p}=0.271$ & $\mathrm{p}=0.08$ & $\mathrm{p}=0.04$ \\
\hline
\end{tabular}


SUVtumor (58.02\% vs $78.59 \%$ ). Regarding percentage change in SUVnode no difference was found (Table 3). Regarding the percentage change in SUVnode, no differences were found.

\subsection{Pathological Response}

Sixteen patients underwent surgical resection. Pathological findings were: G1 (absence of residual tumor): 3.6\%, G2 (rare residual tumor over a lot of fibrosis): $5.4 \%$, G3 (predominate fibrosis over residual tumor): $1.8 \%$, G4 (more residual tumor than fibrosis) 12.5\%, G5 (absence of response): $3.6 \%$ and 1 unknown. Six patients had been treated with CTX and 10 patients with RCTX. Nine patients were adenocarcinoma and 7 patients squamous carcinoma. Most of them were gastroesophageal junction tumors (13/16). According to the degree of tumor response, no differences were observed between PET/CT1 and PET/CT2. However, we observed differences in SUVnode of PET/CT1 between tumors with a low degree or high degree of response to treatment $(p=0.016)$.

\subsection{Overall Survival and Outcomes}

Mean overall survival time was 24.18 months; 11.82 months in patients with persistence on PET/CT2 and 29.27 months in patients without persistence on PET/CT2. Patients who underwent surgery were excluded.

On univariate analysis, the overall survival rate was related to the percentage of change in the SUVtumor and SUVnode. The group of $\geq 75 \%$ of change SUVtumor showed a median survival of 37.32 months (IC: $95 \%=49.93-24.70$ ) and the group of $<75 \%$ of change SUVtumor showed a median survival of 18.39 months (IC: $95 \%=25.14-11.65)(\mathrm{p}=0.04$; Figure 2). However, the group of $\geq 75 \%$ change SUVnode showed a median survival of 20.56 months (IC: $95 \%=$ 25.56 - 14.57) and the group of $<75 \%$ change SUVnode showed a median survival of 17.24 months (IC: $95 \%=27.60-60.87)(p=0.14$ : Figure 2).

Finally, we compared differences in SUVmax values of the esophageal tumor and lymph nodes between PET/CT1 and PET/CT2 according to the pattern of relapse. In patients with metastatic relapse, a significant difference was found regarding the percentage change in SUVnode ( $94.63 \%$ vs. $74.09 \%)$.

\section{Discussion}

Currently, the value of FDG-PET/CT in treatment response assessment of EC is not clear, above all after treatment with RCTX. Some studies indicate PET-CT is

Table 3. Comparison between treatments received on PET/CT imaging.

\begin{tabular}{|c|c|c|c|c|c|c|c|}
\hline & & $\begin{array}{l}\text { Pre-SUV } \\
\text { tumor }\end{array}$ & $\begin{array}{l}\text { Post-SUV } \\
\text { tumor }\end{array}$ & $\begin{array}{l}\text { \% change } \\
\text { SUV tumor }\end{array}$ & $\begin{array}{l}\text { Pre-SUV } \\
\text { node }\end{array}$ & $\begin{array}{l}\text { Post-SUV } \\
\text { node }\end{array}$ & $\begin{array}{l}\text { \% change } \\
\text { SUV node }\end{array}$ \\
\hline \multirow{3}{*}{$\begin{array}{l}\text { Radio/chemotherapy } \\
\qquad(\mathrm{n}=47)\end{array}$} & Persistence $(+)$ & $11.07 \pm 3.39$ & $4.37 \pm 2.63$ & $58.02 \% \pm 26.53 \%$ & $5.09 \pm 3.39$ & $2.55 \pm 3.31$ & $59.91 \% \pm 47.05 \%$ \\
\hline & Persistence (-) & $11.54 \pm 5.44$ & $2.44 \pm 2.76$ & $78.59 \% \pm 26.25 \%$ & $3.69 \pm 4.11$ & $0.42 \pm 1.31$ & $89.5 \% \pm 25.12 \%$ \\
\hline & & $\mathrm{p}=0.78$ & $\mathrm{p}=0.04$ & $\mathrm{p}=0.02$ & $\mathrm{p}=0.33$ & $\mathrm{p}=0.07$ & $\mathrm{p}=0.12$ \\
\hline
\end{tabular}




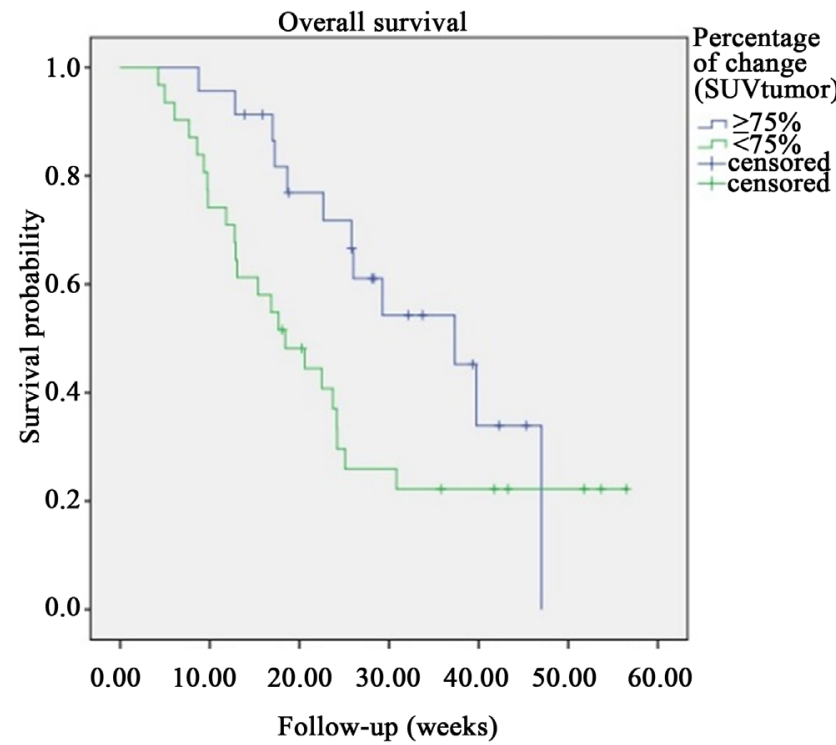

(a)

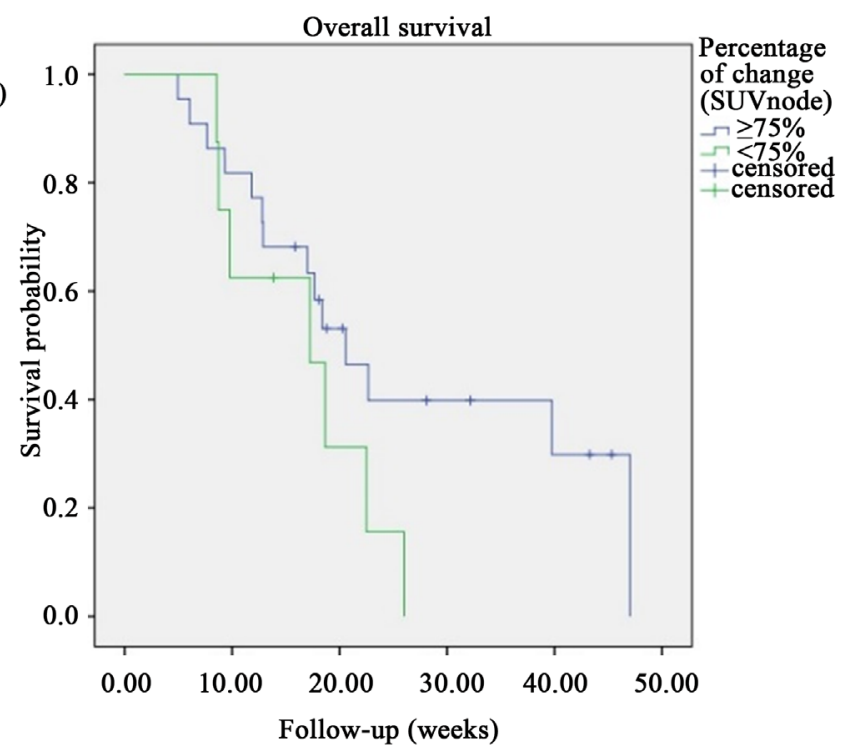

(b)

Figure 2. Overall survival rate for percentage of change SUVtumor (a); $p=0.004$, and for percentage of change SUVnode (b), $p=$ 0.14 .

useful in the reevaluation of treatment [17] and could even be useful in diagnosing a metabolic complete response. The MUNICON trial confirmed the usefulness of an early metabolic evaluation in patients with ADC treated only with neoadjuvant CT [9]. However, other studies have not been able to find a correlation between post-treatment SUV and the pathological response at the time of resection [8]. Even the guidelines differ in their recommendations, $\mathrm{NCCN}^{\bullet}$ guidelines recommend routinely performing a reassessment PET-CT after 4 weeks after completing the treatment, however UPTODATE ${ }^{\circledR}$ recommend not using PET-CT after treatment to select surgical patients until there is no more evidence in this regard.

In addition, although it is currently considered that the optimal treatment of EC is neoadjuvant RCTX or CT followed by surgery, there are studies have questioned the need for esophagectomy after achieving a complete pathological response after neoadjuvant treatment, especially in SCC [7], so non-invasive tests are needed to select patients who respond to initial treatment. A systematic review of 2017 with 697 patients concluded that the available data are contradictory to conclude that FDG-PET has a predictive value in the EC, although there were relevant results that would support its use for the individual therapeutic decision [18].

For this reason, our aim was to analyze the role of FDG-PET/CT in the evaluation of the response to neoadjuvant RCT in the EC. Our results indicate that changes between PET1 and PET2 to predict complete metabolic response. A significant difference was found between the persistence group and the non-persistence group regarding the percentage change in SUVtumor $(72.95 \%$ vs. $54.12 \%$ ) and post-treatment SUVtumor, and regarding the percentage change in SUVnode (89.91\% vs. $59.91 \%)$ and post-treatment SUVnode. This difference 
was maintained between patients receiving RCT, despite the increased uptake that can be caused by inflammation by RTX [19]. In addition, group of patients with $\geq 75 \%$ of change SUVtumor showed a better overall survival than group of patients with $<75 \%$. The selection of the cut-off value of the percentage of tumor change may be important. The range of cut-off values varies widely by different studies, but none have been considered as optimal [20]. Smithers et al. [15] observed the mean percentage change in SUV in the histological responders group was $-56.8 \%$ (SD 29) and in the non-responders $-27.8 \%$ (SD 32.1) $(\mathrm{P}=0.035)$. Brücher et al. [16] concluded that in responders FDG uptake decreased by $72 \%$ $\pm 11 \%$ and in non-responders it decreased by only $42 \% \pm 22 \%$. We use this value in our study because we found the persistence group had a percentage of change in SUVtumor of $72.95 \%$.

There are studies that indicate that a complete pathological response is associated with better survival [21] however, studies only provide conflicting results regarding the utility of FDG-PET images to predict the histopathological response of the primary tumor after neoadjuvant treatment. In addition, optimal PET parameters have not been established to assess the response to treatment, which provides an additional confounding factor. Arnett et al. [8] found no correlation between tumor pathological response and PET findings although, similar to our results, patients with a large tumor reduction after RCT had better results. Otherwise, Cerfolio et al. [22] supported the usefulness of SUVmax as a predictor of pathological response in a study with 86 patients. Similar to our results, they concluded that patients with reduction of $64 \%$ in FDG-PET uptake could predict a complete pathological response, but no relationship with survival was found.

Neoadjuvant treatment could also be a factor to consider. It is known that CTX and RTX affect carbohydrate metabolism in tumor cells. In vitro studies have demonstrated a transient increase in early FDG uptake after exposure to CTX [23], however a similar increase has not been described in vivo. Furthermore, the RTX directly affects the metabolic activity of tumor cells and induces an inflammatory reaction with increased FDG uptake [24], which can mask a metabolic response. Therefore, radiation-induced esophagitis could affect the measurement of FDG uptake by the tumor. However, in our study the difference between the percentage of change between patients with or without persistence of disease was maintained in the group treated with RCTX. In order to minimize these unwanted effects, PET/CT should be carried out 8 - 12 weeks after RCTX.

It is important to note that our study has several limitations, including its retrospective nature and its analysis of a small number of patients with heterogeneous characteristics. Also, most of our patients were SCC and were treated with RCTX without surgery, because they had a histopathologically confirmed complete response. This was caused because the patients were systematically collected from the usual clinical practice, without attending to the histology or neoadjuvant treatment received. Therefore, results cannot be compared with other studies in which patients were subsequently operated. In our hospital, pa- 
tients were selected for the high percentage of PET-CT response and for the clinical information of other tests such as endoscopy or biopsy of the suspicious area.

Another limitation might be the method of metabolic uptake measurements. According to Vallbohmer et al. [25] many factors influence SUV measurements, such as size, measurement duration, plasma glucose concentration, recovery coefficient, partial volume and ROI selection. Other parameters than SUVmax could have been analyzed as MTV (metabolic tumorvolumen), TLG (total lesion glycolysis) or SUVmean, but our patients and their PET-CT were collected from the usual clinical practice, and SUVmax is the value usually used in our hospital.

\section{Conclusion}

In conclusion, our findings indicate that PET/CT imaging might be a standard component of the reevaluation of all locally advanced EC patients, especially to identify patients with or without persistence of tumor disease. The present study provides more evidence of the ability of changes between PET/CT1 vs. PET/CT2 to evaluate tumor response after RCTX. In the future, changes produced between both PET/CTs could be used to decide which patients should undergo surgery. More studies are needed to evaluate post-treatment changes in PET/CT in EC.

\section{Support/Funding}

No Financial Support/Funding Statement.

\section{Conflicts of Interest}

The authors declare no conflicts of interest regarding the publication of this paper.

\section{References}

[1] Pennathur, A., Gibson, M.K., Jobe, B.A. and Luketich, J.D. (2013) Oesophageal Carcinoma. The Lancet, 381, 400-412. https://doi.org/10.1016/S0140-6736(12)60643-6

[2] Sjoquist, K.M., Burmeister, B.H., Smithers, B.M., et al. (2011) Survival after Neoadjuvant Chemotherapy or Chemoradiotherapy for Resectable Oesophageal Carcinoma: An Updated Meta-Analysis. The Lancet Oncology, 12, 681-692. https://doi.org/10.1016/S1470-2045(11)70142-5

[3] Shapiro, J., van Lanschot, J.J.B., Hulshof, M., et al. (2015) Neoadjuvant Chemoradiotherapy plus Surgery versus Surgery Alone for Oesophageal or Junctional Cancer (CROSS): Long-Term Results of a Randomised Controlled Trial. The Lancet Oncology, 16, 1090-1098. https://doi.org/10.1016/S1470-2045(15)00040-6

[4] Stahl, M., Stuschke, M., Lehmann, N., et al. (2005) Chemoradiation with and without Surgery in Patients with Locally Advanced Squamous Cell Carcinoma of the Esophagus. Journal of Clinical Oncology, 23, 2310-2317.

https://doi.org/10.1200/JCO.2005.00.034

[5] Bedenne, L., Michel, P., Bouche, O., et al. (2007) Chemoradiation Followed by Sur- 
gery Compared with Chemoradiation Alone in Squamous Cancer of the Esophagus: FFCD 9102. Journal of Clinical Oncology, 25, 1160-1168.

https://doi.org/10.1200/JCO.2005.04.7118

[6] Bruzzi, J.F., Swisher, S.G., Truong, M.T., et al. (2007) Detection of Interval Distant Metastases: Clinical Utility of Integrated CT-PET Imaging in Patients with Esophageal Carcinoma after Neoadjuvant Therapy. Cancer, 109, 125-134. https://doi.org/10.1002/cncr.22397

[7] Monjazeb, A.M., Riedlinger, G., Aklilu, M., et al. (2010) Outcomes of Patients with Esophageal Cancer Staged with [(1)(8)F]fluorodeoxyglucose Positron Emission Tomography (FDG-PET): Can Postchemoradiotherapy FDG-PET Predict the Utility of Resection? Journal of Clinical Oncology, 28, 4714-4721.

https://doi.org/10.1200/JCO.2010.30.7702

[8] Arnett, M.K., Macintosh, E.M., James, S.E., Nathan, M.A., Shen, K.R., Ravi, K., Neben Wittich, M.A., Haddock, M.G. and Hallemeier, C.L. (2017) Utility of (18)F-FDG PET for Predicting Histopathologic Response in Esophageal Carcinoma Following Chemoradiation. Journal of Thoracic Oncology, 12, 121-128. https://doi.org/10.1016/j.jtho.2016.08.136

[9] Lordick, F., Ott, K., Krause, B.J., et al. (2007) PET to Assess Early Metabolic Response and to Guide Treatment of Adenocarcinoma of the Oesophagogastric Junction: The MUNICON Phase II Trial. The Lancet Oncology, 8, 797-805. https://doi.org/10.1016/S1470-2045(07)70244-9

[10] Jimenez-Jimenez, E., Mateos, P., Aymar, N., et al. (2018) Radiotherapy Volume Delineation Using 18F-FDG-PET/CT Modifies Gross Node Volume in Patients with Oesophageal Cancer. Clinical and Translational Oncology, 20, 1460-1466. https://doi.org/10.1007/s12094-018-1879-3

[11] Leong, T., Everitt, C., Yuen, K., et al. (2006) A Prospective Study to Evaluate the Impact of FDG-PET on CT-Based Radiotherapy Treatment Planning for Oesophageal Cancer. Radiotherapy \& Oncology, 78, 254-261. https://doi.org/10.1016/j.radonc.2006.02.014

[12] Matzinger, O., Gerber, E., Bernstein, Z., et al. (2009) EORTC-ROG Expert Opinion: Radiotherapy Volume and Treatment Guidelines for Neoadjuvant Radiation of Adenocarcinomas of the Gastroesophageal Junction and the Stomach. Radiotherapy \& Oncology, 92, 164-175. https://doi.org/10.1016/j.radonc.2009.03.018

[13] Mandard, A.M., Dalibard, F., Mandard, J.C., et al. (1994) Pathologic Assessment of Tumor Regression after Preoperative Chemoradiotherapy of Esophageal Carcinoma. Clinicopathologic Correlations. Cancer, 73, 2680-2686.

https://doi.org/10.1002/1097-0142(19940601)73:11<2680::AID-CNCR2820731105> 3.0.CO;2-C

[14] Jimenez-Jimenez, E., Mateos, P., Ortiz, I., et al. (2019) Nodal FDG-PET/CT Uptake Influences Outcome and Relapse Location among Esophageal Cancer Patients Submitted to Chemotherapy or Radiochemotherapy. Clinical and Translational Oncology, 21, 1159-1167. https://doi.org/10.1007/s12094-019-02038-6

[15] Smithers, B.M., Couper, G.C., Thomas, J.M., et al. (2008) Positron Emission Tomography and Pathological Evidence of Response to Neoadjuvant Therapy in Adenocarcinoma of the Esophagus. Diseases of the Esophagus, 21, 151-158. https://doi.org/10.1111/j.1442-2050.2007.00732.x

[16] Brucher, B.L., Weber, W., Bauer, M., et al. (2001) Neoadjuvant Therapy of Esophageal Squamous Cell Carcinoma: Response Evaluation by Positron Emission Tomography. Annals of Surgery, 233, 300-309. 
https://doi.org/10.1097/00000658-200103000-00002

[17] Brucher, B.L., Swisher, S.G., Konigsrainer, A., et al. (2009) Response to Preoperative Therapy in Upper Gastrointestinal Cancers. Annals of Surgical Oncology, 16, 878-886. https://doi.org/10.1245/s10434-009-0315-X

[18] Cremonesi, M., Garibaldi, C., Timmerman, R., et al. (2017) Interim (18)F-FDGPET/CT during Chemo-Radiotherapy in the Management of Oesophageal Cancer Patients. A Systematic Review. Radiotherapy and Oncology, 125, 200-212. https://doi.org/10.1016/j.radonc.2017.09.022

[19] Culverwell, A.D., Scarsbrook, A.F. and Chowdhury, F.U. (2011) False-Positive Uptake on 2-[(1)(8)F]-fluoro-2-deoxy-D-glucose (FDG) Positron-Emission Tomography/Computed Tomography (PET/CT) in Oncological Imaging. Clinical Radiology, 66, 366-382. https://doi.org/10.1016/j.crad.2010.12.004

[20] Malik, V., Lucey, J.A., Duffy, G.J., et al. (2010) Early Repeated 18F-FDG PET Scans during Neoadjuvant Chemoradiation Fail to Predict Histopathologic Response or Survival Benefit in Adenocarcinoma of the Esophagus. Journal of Nuclear Medicine, 51, 1863-1869. https://doi.org/10.2967/jnumed.110.079566

[21] Castoro, C., Scarpa, M., Cagol, M., et al. (2013) Complete Clinical Response after Neoadjuvant Chemoradiotherapy for Squamous Cell Cancer of the Thoracic Oesophagus: Is Surgery Always Necessary? Journal of Gastrointestinal Surgery, 17, 1375-1381. https://doi.org/10.1007/s11605-013-2269-3

[22] Cerfolio, R.J., Bryant, A.S., Talati, A.A., et al. (2009) Change in Maximum Standardized Uptake Value on Repeat Positron Emission Tomography after Chemoradiotherapy in Patients with Esophageal Cancer Identifies Complete Responders. The Journal of Thoracic and Cardiovascular Surgery, 137, 605-609. https://doi.org/10.1016/j.jtcvs.2008.11.016

[23] Haberkorn, U., Morr, I., Oberdorfer, F., et al. (1994) Fluorodeoxyglucose Uptake in Vitro: Aspects of Method and Effects of Treatment with Gemcitabine. Journal of Nuclear Medicine, 35, 1842-1850.

[24] Schiepers, C., Haustermans, K., Geboes, K., et al. (1999) The Effect of Preoperative Radiation Therapy on Glucose Utilization and Cell Kinetics in Patients with Primary Rectal Carcinoma. Cancer, 85, 803-811. https://doi.org/10.1002/(SICI)1097-0142(19990215)85:4<803::AID-CNCR7>3.0.CO; $\underline{2-\mathrm{T}}$

[25] Vallbohmer, D., Holscher, A.H., Dietlein, M., et al. (2009) [18F]-Fluorodeoxyglucose-Positron Emission Tomography for the Assessment of Histopathologic Response and Prognosis after Completion of Neoadjuvant Chemoradiation in Esophageal Cancer. Annals of Surgery, 250, 888-894. https://doi.org/10.1097/SLA.0b013e3181bc9c0d 